University of Wollongong

Research Online

Faculty of Social Sciences - Papers (Archive) Faculty of Arts, Social Sciences \& Humanities

2005

Interactions between genetic and environmental factors determine direction of population lateralization

Chao Deng

University of Wollongong, chao@uow.edu.au

Follow this and additional works at: https://ro.uow.edu.au/sspapers

Part of the Education Commons, and the Social and Behavioral Sciences Commons

Research Online is the open access institutional repository for the University of Wollongong. For further information contact the UOW Library: research-pubs@uow.edu.au 


\title{
Interactions between genetic and environmental factors determine direction of population lateralization
}

\author{
Abstract \\ Direction of the embyro's head rotation is determined by asymmetrical expression of several genes (such \\ as shh, nodal, lefty, and fgf8) in hensen's node. this genetically determined head-turning bias provides a \\ base for light-aligned population lateralization in chicks, in which the direction of the lateralization is \\ determined by genetic factors and the degree of the lateralization is determined by environmental factors.

\section{Keywords} \\ population, direction, determine, lateralization, factors, interactions, environmental, genetic, between

\section{Disciplines} \\ Education | Social and Behavioral Sciences

\section{Publication Details} \\ Deng, C. (2005). Interactions between genetic and environmental factors determine direction of \\ population lateralization. Behavioural and Brain Sciences: an International Journal of Current Research \\ anf Theory with Open Peer Commentary, 28 (4), 598.
}


Commentary/Vallortigara \& Rogers: Survival with an asymmetrical brain

\section{Interactions between genetic and environmental factors determine direction of population lateralization}

\section{Chao Deng}

Department of Biomedical Science, University of Wollongong, Wollongong,

NSW 2522, Australia. chao@uow.edu.au

Abstract: Direction of the embyro's head rotation is determined by asymmetrical expression of several genes (such as shh, Nodal, lefty, and FGF8) in Hensen's node. This genetically determined head-turning bias provides a base for light-aligned population lateralization in chicks, in which the $d i$ rection of the lateralization is determined by genetic factors and the degree of the lateralization is determined by environmental factors.

Using the concept of an "evolutionarily stable strategy (ESS)/frequency-dependent selection" hypothesis, and "social selective theory," Vallortigara \& Rogers (V\&R) explain well the evolution of lateralization of avoidance-aggressive behavior or the lateral biases in prey-predator interactions at the population-level. However, other forms of lateralization might not fit this theory. One reason is that not all behavioral lateralization (e.g., lateralization of song control in birds and other cognitive functions) at the population level is predictable for other organisms; therefore, the population lateralization of these behaviors could not be simply said to be more disadvantageous as V\&R suggest in their example about the lateralization involved in prey-predator interactions.

The authors have summarized the developmental mechanisms by which lateralization is aligned at the population level. Taking the development of visual lateralization in the chick and pigeon as examples, lateralized light stimulation of right eye during the later stages of incubation induces the motor, visual, and cognitive lateralization at the population level (Casey \& Martino 2000; Rogers 1982; Rogers \& Andrew 2002). This results because, during this period, the embryo of these birds turns its head against the left side, so that the left eye is occluded by the body and the right eye is positioned next to the air sac. Recent studies have shown that a left-right asymmetry in position of the visceral organ and direction of the embyro's head rotation are determined by asymmetrical expression of sonic hedgehog (Shh), Nodal, lefty, and FGF8 genes in and around the chicken organizer (Hensen's node) during and after the gastrulation stage of embryonic development (Boettger et al. 1999; Levin et al. 1995; Meyers \& Martin 1999; Schier 2003). Therefore, population lateralization aligned by asymmetrically light stimulation during the embryonic development is based on genetically determined head turning.

Chicks hatched from dark-incubated eggs showed asymmetry in using the left or right monocular field (Deng \& Rogers 2002a; Vallortigara et al. 2001), but they did not present a population lateralization on tested behaviors (attack and copulation responses and grain-pebble categorization). V\&R state that genetic expression determines the lateralization at the individual level, and light exposure aligns the direction of lateralization at the population level. However, I argue that if the head turning direction has been determined by the genetic expression, only the right eye could be stimulated visually while the incubated eggs were exposed to the light. It is certain that light exposure prior to hatch aligns the various forms of visual lateralization at a population level, but the direction of the lateralization has already been determined by genetically determined head position. In the laboratory, we have observed that nearly all embryos turn their heads to the left side; only in very rare cases is the embryo's head turned to the other side. Therefore, in the natural condition, there are only two possibilities for the light-induced lateralization in chicks or pigeons: no lateralization (dark incubation) or lateralization aligned with right-eye stimulation. This suggests that the visual lateralization in the chick (as mentioned above) is caused by interaction between genetic and environmental factors.

In the laboratory condition, we have found that visual lateralization aligned with left eye (exposure to light) could be achieved at a population level by manipulating the embryo's head position (Deng \& Rogers 2002b). By withdrawing the embryo's head from the egg on day E19 of incubation and applying a black patch to the right eye, we can expose only the left eye to light. Under this experimental condition, the visual lateralization is observed to be in a reversed direction from the normal right-eye lateralization. This result suggests that in the chick (perhaps also in pigeons) visual lateralization aligned with light exposure of the left eye is physiologically possible. However, this option does not exist in the natural condition since the genetic factors have determined the embryo's head turning only to the left. In addition, all studies done by manipulating various steroid hormones (testosterone, estrogen, and corticosterone) have shown that the hormone treatment can only reduce the degree of visual lateralization but cannot change the direction of lateralization (Rogers \& Deng 2005; Rogers \& Rajendra 1993; Schwarz \& Rogers 1992). All of these manipulating experiments support the crucial role of genetic and environmental interaction: genetic factors determining the direction of lateralization, and environmental factors determining the degree of lateralization. Therefore, in the natural condition, no matter how large the changes or whether the animal is actively manipulated as proposed by $V \& R$, if the changes are only in hormone level or light stimulation but not in head-turning direction (determined by genetic factors), the effect will be only on the degree of lateralization and not on the direction of lateralization.

It is very interesting that several genes (including Nodal and Lefty2), which play key roles in controlling the direction of embryo head turning, are evolutionarily conserved in vertebrate (Przemeck et al. 2003). For example, Nodal is expressed asymmetrically in all vertebrates (Stern 2002). Therefore the headturning bias should have evolved very early and been conserved. In fact, turn bias of the embryonic head has also been observed in the human embryo and newborns (Ververs et al. 1994), which has suggested that this head-turning bias induces right-sided lateralization of perception and action at the population level in humans (Güntürkün 2003a). This raises a question, for those forms of population lateralization (e.g., lateralization of song control or other cognitive function) that could not be easily explained by the ESS/ "frequency-dependent selection" hypothesis, of whether their direction of lateralization at the population level is determined by genetic and environmental interactions.

\section{Rethinking brain asymmetries in humans}

\author{
Bianca Dräger, Caterina Breitenstein, and Stefan Knecht \\ Department of Neurology, University of Münster, 48149 Münster, Germany. \\ dragerb@uni-muenster.de caterina.breitenstein@uni-muenster.de \\ knecht@uni-muenster.de \\ http://neurologie.uni-muenster.de/ger/mitarbeiter/breitenstein/ \\ http://neurolog.uni-muenster.de/cms/front_content.php?idcat=34
}

Abstract: Similar to directional asymmetries in animals, language lateralization in humans follows a bimodal distribution. A majority of individuals are lateralized to the left and a minority of individuals are lateralized to the right side of the brain. However, a biological advantage for either lateralization is lacking. The scenario outlined by Vallortigara \& Rogers (V\&R) suggests that language lateralization in humans is not specific to language or human speciation but simply follows an evolutionarily conserved organizational principle of the brain.

Vallortigara \& Rogers (V\&R) show that brain asymmetries are a species independent, ubiquitous phenomenon. They focus on discussing the causes for population asymmetries in various nonhuman species and give evidence that directional brain asymmetries are in some cases behaviorally adaptive and in other cases without any perceptible advantage. Even though they intentionally leave out human asymmetries, V\&R's contribution reframes the discussion of brain lateralization in humans. The discussion initiated in the target article provides new vantage points for an old debate. 